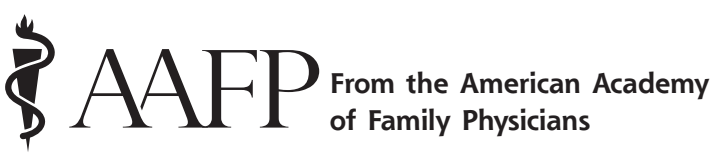

Ann Fam Med 2017;15:285-286. https://doi.org/10.1370/afm.2079.

For this issue, the AAFP is pleased to use this space for a paper by Family Medicine for America's Health, a collaboration of the Academy and seven other national family medicine organizations.

\section{VISION FOR A PRINCIPLED REDESIGN OF HEALTH INFORMATION TECHNOLOGY}

The mission for Family Medicine for America's Health (FMAHealth) is to help people live healthier. To support this, a goal of the FMAHealth Technology Tactic Team is to envision a future state that involves the principled redesign and implementation of health information technology (IT) that optimally supports the health and health care of the US populace.

\section{Problem Statement}

Compared to other nations, the US health care system offers higher cost, lower quality health care to its people. ${ }^{1}$ The "Triple Aim" goals of better health, better quality, and better value have been prominent features of the national, state, and local policy work to realign the health care system, but the path to success is long and must navigate complex social, cultural, political, and economic barriers.

To support and promote the health of our nation, health professionals must develop meaningful relationships with the people, families, and communities they serve. Technology has great potential to help foster connections and relationships among health care professionals, individuals, and communities, and to be a catalyst instead of the barrier it frequently is today. In addition to putting up barriers to achieving the Triple Aim, the poor usability and utility is resulting in health IT contributing to the growing problem of physician burnout.

\section{Charge}

No profession in US health care is better situated to achieve the Triple Aim than empowered, informed primary care doctors and their teams. Primary care physicians are healers, leaders, and partners, and it is through partnership with specialist leaders, including in the field of public health, and others in the community that improved health and health care can be achieved. We believe that new types of information and new kinds of technology are needed.

\section{Process and Guiding Principles}

The FMAHealth Technology Tactic Team hosted a 1-day Visioning Summit and invited a small group of technology and health IT leaders, innovators, and visionaries to engage in a process whereby we would move beyond present-day constraints to envision a future health care system where primary care has a central role in making meaningful improvements in the health of the individuals, communities, and populations that they serve, and in which health IT is a critical enabler of that work.

The guiding principles for this work included agreement that the vision needed to be technologically feasible and internally consistent, and the design principles developed needed to (1) foster connections among health care professionals, including the individuals and communities they serve, and the environment in which people live ${ }_{i}(2)$ accumulate and analyze data that can support these connections and address the needs of population health; and (3) promote appropriate payment for health care.

Additionally, the group explicitly recognized that technology was not "a fix" for what everyone took to be a "broken" health care system. Thus, the group approached its work with a continued recognition that technology was just one part of a multipart, complex, and evolving health system, and solutions would emerge from this system, and need to change at the same time.

\section{Vision}

A vision is an idea that describes a future state and provides a sense of aspiration. Our group developed an overarching vision and a series of "vision attributes," which will guide the Technology Tactic Team and others to identify the capabilities required to deliver on those attributes.

\section{Overarching Vision}

In 10 years, we will have a sustainable health care system that plays a central role in improving the health of the US population. Individuals and the people who take care of them will understand their health, know when their health is deteriorating, and know how to get help (have a usual source of care). Technology will facilitate an ease of knowing, allowing individuals and their health care professionals to have a comprehensive view of their health, which includes individual, community, and environmental aspects of health, and to use this information in developing and executing personalized care plans. We call this Precision Population Health Management, which also includes leveraging technology to identify and decrease health disparities. This monitoring would be both at a high 
level, such as a "worry score," and more granularly based on the population's (or individual's) health issues and socioeconomic situation. Primary care would be equipped to manage and interact with data from the community and individuals to perform the health monitoring and to establish interventions at the community and individual levels. The practice would join with community resources and individuals to effect improvements in the health of the population; we call these activities joining tactics. Use of technology will be frictionless to the physician and other health care professionals, and will support them in their work and be enjoyable to use.

\section{Vision Attributes}

The health IT in use does not support this vision. We provide a list of expectations we believe should form a national priority to close the gap in current health IT.

We expect that in

- 1 year:

- Data visualization technologies, which make it easy for the clinician to see patterns and make insight, will emerge to support health-related decisions and actions

- Technologies will emerge to remove barriers to doctor-patient communication, and begin to create greater connection, transparency, and trust that is cognizant of patient diversity and needs

- Technologies will begin to efficiently integrate sources of patient-generated health data and reported quality data into the record

- We will see new technologies and new roles for technology that enable health system redesign and improvement, while supporting comprehensive payment models that focus on care delivery and health

- 3 years:

- Technology that will create transparency, accuracy, and actionable health-related data will be widely available

- Technology providing easy ways to natively support healthy behaviors, such as improved diet and exercise, and use of safety equipment (i.e. seatbelt, helmet, etc.), will be widely available

- Technology to support more efficient and more reliable learning in our health system (eg, rapid assessment of the effectiveness of a flu vaccine) will be widely available

- Technology will integrate evidence-based medicine into the workflow at the point of care and will rapidly update embedded knowledge as new knowledge is discovered

- Technology that enables every person in the United States to have their own health record that summarizes the medical, social, emotional, and environmental factors relevant to them will be in common use

- Technology will support value-based care and payment to enable achievement of the Triple Aim

- 5 years:

- Technology will enable task interoperability (ie, sharing of tasks between individuals, viewing status of tasks assigned to others, and delegating started tasks), allowing doctors and patients to know what is happening in parts of the care process that may be obscured from his/her view

- Technology will enable individuals and communities to aggregate data to meaningfully compute health indicators so that emerging problems are identified early, and health and wellbeing are improved

- 7 years:

- We will effectively use technology to deliver meaningful and relevant health-related information at the right time in a way that is "frictionless" and supports bringing the joy back to the practice of medicine

- We will effectively use technology to bridge care gaps and reduce health care disparities

- 10 years:

- Technology will fully support primary care doctors to be leaders, partners, and advocates for the health of all; ultimately technology will allow patients to be in control of their health

Steven E. Waldren, $M D_{i}$ Deborab J. Coben, $P b D_{i} J a c o b M$. Reider, $M D_{i}$ Jewell P. Carr, MDi Ciarán A. DellaFera, $M D$ Family Medicine for America's Health (Drs. Waldren, Cohen, Reider, Carr, and DellaFera), American Academy of Family Physicians (Dr. Waldren); Oregon Health \& Science University Department of Family Medicine (Dr. Cohen), Carolinas HealthCare System Department of Family Medicine (Dr. Carr), and Greater Lawrence Family Health Center (Dr. DellaFera)

\section{References}

1. Organisation for Economic Co-operation and Development. Health Statistics 2015. http://www.oecd.org/els/health-systems/health-data. htm. Accessed June 3, 2016. 